\title{
Scientific Realism, the Semantic View and Evolutionary Biology
}

\author{
Fabio Sterpetti \\ Department of Philosophy, Sapienza University of Rome, Italy \\ fabio.sterpetti@uniromal.it
}

\begin{abstract}
The semantic view of theories is normally considered to be an account of theories congenial to Scientific Realism. Recently, it has been argued that Ontic Structural Realism could be fruitfully applied, in combination with the semantic view, to some of the philosophical issues peculiarly related to biology. Given the central role that models have in the semantic view, and the relevance that mathematics has in the definition of the concept of model, the focus will be on population genetics, which is one of the most mathematized areas in biology. We will analyse some of the difficulties which arise when trying to use Ontic Structural Realism to account for evolutionary biology.
\end{abstract}

Keywords: scientific realism; structural realism; semantic view of theories; evolutionary biology; population genetics; models.

\section{Introduction}

Recently, Steven French [1] has claimed that Ontic Structural Realism (OSR), a position normally held by philosophers interested in metaphysically accounting for physical theories, may be, in combination with the semantic view of theories, fruitfully adopted also to account for biological theories, especially population genetics. The present work is aimed at assessing whether this proposal hits the mark or not.

This paper will firstly briefly present the context in which OSR has been developed, and what is the main problem that this position has to face (sec. 2); then, it will briefly present the semantic view and three of the main problems that this position has to face (sec. 3); then, the paper will take into account two different possible responses to the main problem of structuralism, i.e. Psillos' and French's responses, and it will try to underline the difficulties of each position (sec. 4). Finally, the paper will focus on one of the examples given by French to illustrate his proposal of adopting structuralism in biology, i.e. Price's Equation, and it will try to spell out the difficulties of supporting French's claims on the metaphysical significance of Price's Equation for population genetics (sec. 5). 


\section{Scientific Structural Realism}

\subsection{Scientific Realism}

Scientific Realism (SR) can be briefly described as the claim that our best scientific theories are true. As Saatsi and Vickers state: "scientific realists seek to establish a link between theoretical truth and predictive success" [2, p. 29]. SR is based on a two step strategy [3]: 1) infer from the empirical success the truth of the scientific theories; 2 ) infer from the truth of the successful scientific theories the existence of those entities which appear in such theories. So, the claim that theories are able to refer to such existing entities is justified from their empirical success, while this very same ability explains their predictive empirical success.

\section{$2.2 \quad$ Truth}

The concept of truth is central for SR. For example, Giere states that: "virtually every characterization of scientific realism I have ever seen has been framed in terms of truth" [4, p. 154]. The most shared view of truth among the realists is that of truth as correspondence. For example, Sankey states that: "correspondence theories which treat truth as a relation between language and reality are the only theories of truth compatible with realism" [5, p. 17].

Given that the crucial element in order to claim for the truth of a theory is the confirmation of such theory, and that confirmation doesn't allow to discriminate between the different parts of the theory which has been confirmed, and that theories usually contain theoretical terms, i.e. terms which refer to some unobservables, realists believe in the existence of the theoretical terms postulated by the confirmed theory.

\subsection{The No Miracle Argument}

The main argument to support SR is the No Miracle Argument (NMA). Putnam formulated the NMA as follows: "The positive argument for realism is that it is the only philosophy that does not make the success of science a miracle" [6, p. 73]. The central idea of the NMA is that the truth of a scientific theory is the best, or the only scientifically acceptable, explanation of its empirical success. The problem is that, given the traditionally accepted realist view of truth, claiming that the success of a theory is due to its being true would imply that such theory should not be radically modified over time or ever considered false.

\subsection{The Pessimistic Meta-Induction}

But the history of science seems not to allow us to support such a claim. The Pessimistic Meta-Induction (PMI), firstly developed by Laudan [7], can be briefly summarized as follows: 
(1) The historical record reveals that past theories which were successful turned out to have been false.

(2) So, our present scientific theories, although successful, will probably turn out to be false.

(3) Therefore, we should not believe our best present theories. ${ }^{1}$

To face the PMI different strategies have been developed by the realists. Many of them try to show that despite the theory shift, something is retained from one theory to another, and that it is just such 'something' that the realist is committed to.

\subsection{Scientific Structural Realism}

The most credited position in this realist line of reasoning is Scientific Structural Realism (SSR). French states that SSR has been developed exactly "to overcome the socalled Pessimistic Meta-Induction, which presents the realist with the problem of accommodating the apparent historical fact of often-dramatic ontological change in science" [9, p. 165]. Even if SSR does not rely on the NMA, it is normally considered to be able to support the intuition at the origin of the NMA, i.e. that there is a deep correlation between success and truth. ${ }^{2}$ SSR is articulated in two main positions: Epistemic Structural Realism (ESR), which claims that we can be realist only about the mathematical structure of our theories [11], and OSR, which claims that structure is all there is [12]. ${ }^{3}$ So, what is thought not to change during the theory shift by SSR is the mathematical structure of the theories. For example, Sneed says that "structuralists see the mathematical structures associated with a theory to be much more 'essential' features of the theory than the claims it makes. The claims may change with the historical development of the theory, but the mathematical apparatus remains the same" $[14$, p. 351].

\subsection{Scientific Structural Realism and the Semantic View}

The focus on the mathematical structures of the theories makes clear why those who support SSR usually support the semantic view of theories [15], the view according to which a theory is the class of its models. For example, Ladyman states that the "semantic' or 'model-theoretic' approach to theories, [...], is particularly appropriate for the structural realist" [12, p. 417], and suggests that "structural realists adopt Giere's account of theoretical commitment: to accept a theory means believing that the world

\footnotetext{
1 [8, p. 804].

2 Cf. [10, p. 45]: "structural realism is supposed to be realist enough to take account of the nomiracles argument."

3 See [13] for a survey on SSR. For a definition of structure, cf., e.g., Ibidem, p. 229: “A structure $S$ consists of (a) a non-empty set $U$ of objects, which form the domain of the structure, and (b) a non-empty indexed set $R$ (i.e. an ordered list) of relations on $U$, where $R$ can also contain one-place relations."
} 
is similar or isomorphic to one of its models" [16, p. 185]. Indeed, the way in which models are intended in the semantic view is the same in which models are intended in metamathematics. Thus, they are mathematical structures. To see this is particularly easy: the relation of isomorphism, which is claimed to hold among the models of a theory by the semanticists, is defined exactly in the same terms in which the relation of isomorphism is defined in model theory. Semanticists look at Tarski as the initiator of the semantic view of theories [17] and explicitly adopt the Tarskian concept of model in their view. For example, Suppes claims that "'the concept of model in the sense of Tarski may be used without distortion and as a fundamental concept' in scientific and mathematical disciplines," and that "'the meaning of the concept of model is the same in mathematics and the empirical sciences';" so, we can conclude that for him "the Tarski concept of a model is a common formal framework for analysis of various uses of models in science and mathematics" [18, pp. S110-S111].

\subsection{Ontic Structural Realism and Mathematics}

If the structuralist supports ESR, she is committed to the indispensable role of mathematics in accounting for theory change in a realist fashion. But if she supports OSR, she is also committed to the existence of the mathematical structures which figure in the theory, and so she has to face the risk of let her position become a full-blood Pythagorean position. Indeed, "Pythagoreanism [...] is the teaching that the ultimate 'natural kinds' in science are those of pure mathematics" [19, p. 60].

This risk that the supporters of OSR have to face, has been labeled by French the 'Collapse Problem' [1]: if the world is isomorphic to theories, and isomorphism can hold only between mathematical structures, then the world is a mathematical structure. Tegmark, for example, "explains the utility of mathematics for describing the physical world as a natural consequence of the fact that the latter is a mathematical structure, and we are simply uncovering this bit by bit. [...]. In other words, our successful theories are not mathematics approximating physics, but mathematics approximating mathematics" [20, p. 107]. In fact, Tegmark argues, "the external reality is" not "described by mathematics, [...] it is mathematics [...]. This corresponds to the 'ontic' version of universal structural realism [...]. We write is rather than corresponds to here, because if two structures are isomorphic, then there is no meaningful sense in which they are not one and the same. From the definition of a mathematical structure [...], it follows that if there is an isomorphism between a mathematical structure and another structure [...], then they are one and the same. If our external physical reality is isomorphic to a mathematical structure, it therefore fits the definition of being a mathematical structure" (Ibidem).

\section{The Semantic View of Theories and Biology}

\subsection{The Semantic View and Evolutionary Biology}

Since the eighties many authors have been supporting the semantic view of theories as the best account of evolutionary biology, and tried to elaborate a semanticist account 
of evolution [21-23]. This view has rapidly become the received view. The reasons for proposing and accepting the semantic view as the best account of biological theories were basically two: 1) the difficulties afflicting the traditional syntactic account of theories; 2) the more specific fact that, given that biology is normally considered to lack general laws from which starting to axiomatize an entire field of research [24], the semantic view seemed to be more adequate to meta-theoretically describe the biological theories, directly presenting a set of their models, instead of trying to axiomatize them.

In what follows, we will firstly describe some of the difficulties which afflict the semantic view in general, and then a specific difficulty related to the attempt of semantically representing the evolutionary processes.

\subsection{Two Main Difficulties of the Semantic View}

The semantic view of theories can be seen as composed by two parts: the first which equates theories and classes of models, the second which defines the relation between such models and the empirical world [16]. Both these parts have been challenged.

Halvorson focuses his criticisms on the first part, and shows that such "first component is a mistake; i.e., a class of models is not the correct mathematical component of a theory" [16, p. 189], because "this view equates theories that are distinct, and it distinguishes theories that are equivalent" (Ibidem, p. 183). In fact, Halvorson shows that there is no good notion of isomorphism between classes of models, and so that the semantic account fails to provide a satisfactory account of the identity of theories. ${ }^{4}$

This is a big problem for the semanticists, because the possibility of clearly identifying a theory is considered essential in order to give a realist account of the theory change which can avoid the PMI. Indeed, as we have seen above, to avoid the PMI has been the principal motivation for the development of SSR. Suppe explicitly states

4 For technical details and examples, see [16]. Here the room suffices just to sketch Halvorson's argument in five points: 1) given that, according to the semantic view, a theory is a class of models, if we have two classes of models, $\mathcal{M}$ and $\mathcal{M}^{\prime}$, under which conditions should we say that they represent the same theory? 2) Semanticists (e.g., Giere, Ladyman, Suppe, van Fraassen) have not offered any sort of explicit definition of the form: (X) $\mathcal{M}$ is the same theory as $\mathcal{M}^{\prime}$ if and only if (iff) ... 3) "Suppe's claim that 'the theories will be equivalent just in case we can prove a representation theorem showing that $\mathcal{M}$ and $\mathcal{M}^{\prime}$ are isomorphic (structurally equivalent)' [...] just pushes the question back one level - we must now ask what it means to say that two classes of models are 'isomorphic' or 'structurally equivalent'" (Halvorson, 2012, p. 190). 4) He then considers the following three proposals for defining the notion of an isomorphism between $\mathcal{M}$ and $\mathcal{M}^{\prime}$ : (a) Equinumerous: $\mathcal{M}$ is the same theory as $\mathcal{M}^{\prime}$ iff $\mathcal{M} \simeq \mathcal{M}^{\prime}$; that is, there is a bijection $F: \mathcal{M} \rightarrow \mathcal{M}^{\prime}$. (b) Pointwise isomorphism of models: $\mathcal{M}$ is the same theory as $\mathcal{M}^{\prime}$, just in case there is a bijection $F: \mathcal{M} \rightarrow \mathcal{M}^{\prime}$ such that each model $m \in \mathcal{M}$ is isomorphic to its paired model $F(m) \in \mathcal{M}^{\prime}$. (c) Identity: $\mathcal{M}$ is the same theory as $\mathcal{M}^{\prime}$, just in case $\mathcal{M}=\mathcal{M}^{\prime}$. 5) Finally, he tests such proposals and shows how they all fail, and thus makes it clear that it is impossible to formulate good identity criteria for theories when they are considered as classes of models. 


\section{PENULTIMATE DRAFT - PLEASE CITE THE PUBLISHED VERSION}

To appear in: Models and Inferences in Science, Ippoliti, E., Sterpetti, F. and Nickles, T. (eds.), Springer.

that the semantic view "is inadequate if it cannot properly individuate theories. Theories undergo development. This has implications for theory individuation," because the semantic view "essentially treats theory development as progression of successive theories," and he adds that he considers "theory individuation issues as make-or-break for any account of theories" [18, pp. S108-S109]. To sum up: there is a deep relation between OSR and the semantic view. The issue of theory individuation is considered to be crucial by the semanticists themselves to determine whether the semantic view is a tenable position or not, and Halvorson's work seriously treats exactly such crucial requisite of the semantic view.

Chakravartty has challenged the second part of the semantic view: given that the semantic view of theories is deeply related to the concept of truth as correspondence, if the semantic view is language independent, it cannot satisfactorily account for a correspondence relation. The problem is the following: how is it possible to state that theories (i.e. classes of models) correspond to the world, if the required realist definition of such a correspondence, e.g. that of Tarski, is relative to (formal) languages, while theories are supposed to be non-linguistic entities by the semanticists? [25]. Thus, in order to qualify the semantic view as being able to satisfy the realist's claims, it seems necessary to presuppose a correspondence relation between the mathematical structure of theories and the structure of the world.

The problem is precisely how to account for such presupposition. To answer the question: "why an abstract structure such as a mathematical model can describe the non abstract physical world?" the realist's response usually "depicts nature as itself a relational structure in precisely the same way that a mathematical object is a structure. On this view, if the mathematical model represents reality, it does so in the sense that it is a picture or copy [...] of the structure that is there" [26, p. 242]. So, the realist seems to subscribe to a sort of substantial correspondence theory of truth and representation. But when she is pressed by the fact that there are different ways of mathematically representing the physical world, the realist cannot do better than "insists that there is an essentially unique privileged way of representing: 'carving nature at the joints'. There is an objective distinction 'in nature' between on the one hand arbitrary or gerrymandered and on the other hand natural divisions" (Ibidem, p. 244). How does she justify such assertion? She doesn't: "It is a postulate" (Ibidem).

\subsection{Gildenhuys' Attack on the Semantic View}

Recently, Gildenhuys has pointed out a difficulty which afflicts the semantic attempt to describe evolutionary biology, more precisely population genetics [27]. In fact, in such approach models are normally described as states in the phase space of the represented systems:

The models picked out are mathematical models of the evolution of states of a given system [...]. This selection is achieved by conceiving of the ideal system as capable of a certain set of states - these states are represented by elements of a certain mathematical space [...]. The variables used in each mathematical model represent various measurable or potentially quantifiable physical magnitudes [...] any particular configuration of 
values for these variables is a state of the system, the state space or 'phase space' being the collection of all possible configurations of the variables. ${ }^{5}$

According to Gildenhuys, this view is inadequate to describe population genetics, because even if philosophers have argued that, "in comparison to the rival syntactic approach to scientific theories," the semantic view "provides a superior framework for presenting population genetics [...], none of these writers has specified the class of mathematical structures that constitutes population genetics or any of its formalisms" [27, p. 274].

Details are not relevant here, what is worth noting is that Gildenhuys not only shows that a clear and complete definition of the 'phase space' of a biological system has never actually been given, but also that it would not be easy to give it for mathematical reasons. Indeed, he focuses on Lloyd's formulation, according to which there are "two main aspects to defining a model. First, the state space must be defined [...]; second, coexistence laws, which describe the structure of the system, and laws of succession, which describe changes in its structure, must be defined" [28, p. 19]. Then, Gildenhuys underlines the challenge "posed by the existence of coefficients in population genetics whose values are set by functions" [27, p. 281].

The problem is that if we try to construct the class of such functions, we get a mathematical indefinite object, and so we are not able to give the phase space we should instead construct to model population genetics in a semanticist fashion. Roughly, the difficulty lays in how to relate the causal relations among the individuals and all the possible different fitness functions deriving by their interactions. Indeed, "by means of functions that feature relative frequency terms as arguments, frequency dependent selection functions capture causal dependencies among the individuals" (Ibidem, p. 282). The problem is that when the character of the causal dependencies among population members varies, "the functions that set fitness values must vary with them. This variation is not merely variation in the values taken by a fixed set of arguments arranged in a fixed functional form. Causal relationships among individuals [...] need not be linear [...]. Equally, they may feature exogenous variables" (Ibidem, pp. 282-283). Now, to see "how fitness functions [...] pose an obstacle to using" the semantic approach to describe population genetics, notice that "alternative fitness functions are inconsistent. Different fitness functions serve as alternative equations for setting the value of a single parameter. They cannot appear side by side, then, in a system of coexistence laws" (Ibidem, p. 284). Moreover, if we try to construct the class of those functions, "there are good reasons to believe that the class of such functions is in fact mathematically indefinite" (Ibidem). Thus, the "charge is that [...] we cannot specify the class of fitness functions" (Ibidem, p. 285) and so that we cannot construct a phase space for population genetics systems.

5 [21, p. 244]. 


\subsection{Longo's View on Phase Space in Biology}

Gildenhuys' criticism may be seen in relation to a wider criticism on the very possibility of giving a phase space when dealing with biological entities, a kind of criticism developed by Giuseppe Longo. Longo identifies the peculiarity of biology with respect to physics exactly in "the mathematical un-predefinability of biological phase space" [29, p. 195]. Indeed, "in contrast to existing physical theories, where phase spaces are pre-given, if one takes organisms and phenotypes as observables in biology, the intended phase spaces need to be analyzed as changing in unpredictable ways through evolution" (Ibidem, p. 189). The fact is that "random events, in biology, do not 'just' modify the (numerical) values of an observable in a pre-given phase space, like in physics [...]. They modify the very phase space" (Ibidem, p. 199). Thus, "one major aspect of biological evolution is the continual change of the pertinent phase space and the unpredictability of these changes" (Ibidem, p. 187). We will not try to assess such criticisms. What is worth noting here is that Gildenhuys and Longo underline the difficulty of giving a definite phase space when dealing with evolutionary theories. If, as many supporters of the semantic approach to evolution maintain, giving the phase space is essential for giving an account of biological theories in accordance to the semantic approach, and the semantic view is the view adopted by the structural realists, then the structural realists should face this kind of difficulty if they want to account for evolutionary biology in structural terms. They may face this difficulty either by giving a definite phase space for the system they want to model, or by showing that giving a phase space is not essential for their approach.

\section{Structural Realism and the Collapse Problem}

\subsection{Facing the Collapse Problem}

The Collapse Problem described above (sec. 2.7) may be better understood considering it from a more general perspective: the pressure that mathematical platonists are doing on scientific realists in order to let the realists accept Mathematical Platonism (MP). For example, Psillos states that philosophy of science "has been a battleground in which a key battle in the philosophy of mathematics is fought $[\ldots]$ indispensability arguments capitalise on the strengths of scientific realism, and in particular of the nomiracles argument [...], in order to suggest that a) the reality of mathematical entities [...] follows from the truth of [...] scientific theories; and b) there are good reasons to take certain theories to be true" [30, p. 63].

The scientific realists may respond to the platonist's pressure in two ways: 1) widening their ontology to accept abstract objects; 2) continuing to rely on causality and trying to avoid a direct commitment to the existence of abstract objects. The first option has been taken, among others, by Psillos. The second option has been taken, among others, by French. In what follows we will describe their approaches and the main difficulties that afflict them. 


\section{PENULTIMATE DRAFT - PLEASE CITE THE PUBLISHED VERSION}

To appear in: Models and Inferences in Science, Ippoliti, E., Sterpetti, F. and Nickles, T. (eds.), Springer.

\subsection{An Analysis of Psillos' Approach}

Psillos' approach is representative of the attempt of 'moving beyond causation' that many realists are pursuing exactly to take into account abstract objects and non-causal (basically, mathematical) explanations [31]. Indeed, classically, SR supported the idea that scientific theories should be taken at face-value. But, "a literal reading of scientific theories implies commitment to a host of entities with a (to say the least) questionable ontic status: numbers, geometrical points, theoretical ideals, models and suchlike" [32, pp. 5-6]. The difficulty of conceiving the reality of the abstracta leads the realist to face what Psillos has called the 'Central Dilemma': "Either theories should be understood literally, but then they are false. Or, they should not be understood literally, but then realism itself is false (at least insofar as it implies that theories should be understood literally)" (Ibidem, p. 6). Indeed, if we commit ourselves to a correspondence view of truth, how could we avoid to take scientific theories to be understood literally? But, if we take a theory to be literally true, then we should believe in the existence of, e.g., numbers. The problem is that numbers are defined by platonists in such a way that they are outside the reach of science, at least to the extent that science is considered to be indispensably related to causality, as the majority of the realists seems still to think. Indeed, the so called 'Eleatic Principle', which can be stated as: "everything that is real makes some causal difference to how the world is" [33, p. 89], has been considered to be able to discriminate what exists, referring to causation, by many realists since a long time.

Moreover, since, as we have seen, ever more realists have started supporting the semantic view of theories, the problem of how to conceive of the nature of models has become central for the realists. In fact, if following the semantic view, "theories are taken to be collections of models," and models are considered as abstract objects, then "theories traffic in abstract entities much more widely than is often assumed:" the claim that models are abstract entities "is meant to imply that (a) they are not concrete and (b) they are not causally efficacious. In this sense, models are like mathematical abstract entities" [32, p. 4]. Thus, models are abstract in the same way in which mathematical entities are claimed to be abstract by the mathematical platonists. Indeed, MP can be briefly described as the claim that mind-independent mathematical abstract entities exist, and abstract entities are normally understood as 'non-spatiotemporally located' and 'causally inert' [34].

To make SR and MP compatible, Psillos adopts an 'explanatory criterion' to determine his realist ontology, explicitly claiming that "something is real if its positing plays an indispensable role in the explanation of well-founded phenomena" [32, $\mathrm{p}$. 15]. He clearly underlines the distance between the explanatory criterion and causality: "This explanatory criterion should not be confused with a causal criterion. It is not a version of the so-called Eleatic principle" (Ibidem).

So, even non causal objects exist, and it seems that their existence could be supported relying only on explanatory considerations. The argument could run something like this: we believe in the existence of what appears to be indispensable in our scientific explanations, abstract entities are indispensible in our best scientific explanations, then abstract objects exist. 
But an explanation could have a great explanatory power and nevertheless be false. So, we could risk to infer the existence of some object which doesn't really (i.e. from a realist perspective) exist. So, how can Psillos deem the explanatoriness and indispensability of an object be reliable means to infer the existence of such indispensable objects? The problem lays in the ambiguity of the way in which Psillos describes the relation between explanations and theories, and between theories and the world.

What Psillos doesn't explicitly state is that an inference from the explanatoriness to the existence could be sound only if one has already accepted the two classical realist assumptions concerning the truth, i.e.: 1) that truth is correspondence to the world, and 2) that our best scientific theories are true because they have empirical success. So, Psillos' argument could be restated as follows: given that we infer the truth of the theories from their success, and that abstract entities are indispensible for the very reaching of such success; if truth is correspondence, the success of a theory is (best) explained by the existence of the objects such theory refers to, then abstract objects exist; a scientific explanation relies on our already selected, i.e. empirically confirmed, best scientific theories; then, if in such explanation an abstract object appears to be indispensable, we can safely commit ourselves to its existence.

In this way, the dangerous equation between explanatoriness and confirmation in order to define ontological matters has been neutralized. This has been possible thanks to the occultation of the link between confirmation and the set of the already accepted theories, and the insertion of the requirement that acceptable explanations from which deriving our ontology can only be drawn from such set of empirically confirmed theories. In such a way, our ontology may appear as based exclusively on the 'explanatory criterion', which Psillos explicitly says that should not be confused with a causal criterion, but such explanatory criterion rests nevertheless upon confirmation, i.e. empirical success, which is at its turn normally intended and explained in causal terms.

So, despite what Psillos explicitly asserts on explanatoriness, confirmation still plays a crucial role for the realists in order to determine the truth of a theory, and causality still plays a crucial role in order to account for the way in which confirmation is obtained and detected. ${ }^{6}$ In fact, in another work (published in the same period) Psillos himself explicitly affirms that the "best explanation (of the instrumental reliability of scientific methodology) is this: the statements of the theory which assert the specific causal connections or mechanisms in virtue of which methods yield successful predictions are approximately true" [35, p. 23]. Thus, it is not easy for a realist to accept $a b s t r a c t a$ and give up causality.

\subsection{The Collapse Problem and the Problem of Representation}

Besides its relation with MP, it is also important to underline the connection between the Collapse Problem and the problem of scientific representation. Indeed, the way in which theories are related to the world is the crucial problem of any kind of realism.

6 At least in the measure in which Psillos doesn't give a different account of how to consider a theory to be empirically confirmed. 


\section{PENULTIMATE DRAFT - PLEASE CITE THE PUBLISHED VERSION}

To appear in: Models and Inferences in Science, Ippoliti, E., Sterpetti, F. and Nickles, T. (eds.), Springer.

For example, Hughes states that "in what sense [...] a physical theory represent the world?" is one of "the key question that philosophers of physics need to address" [36, p. 344].

For SSR, as we have seen, the problem is also related to the problem of the applicability of mathematics: since models are normally intended as not being interpretable as literally true, and models are generally conceived of as mathematical models, the problem of the relation between models and the world amounts to the problem of the relation between mathematics and the world.

This is where the problems described above relative to the semantic view meet the problem afflicting OSR. In fact, being able to solve the problems of the semantic view would imply to be able to distinguish the mathematical from the physical, i.e. to solve the Collapse Problem, because it would amount to be able to identify the right mathematical formulation of a phenomenon among the many which are possible, given that we would be able to state which is the correct representational relation between a mathematical structure and the world, and this could be possible only if the mathematical structures and the world do not coincide, i.e. if they are not the same thing, as Tegmark claims.

The problem is that there is a sort of dilemma here for the realist: either to account for the relation between models and the world she insists on isomorphism, but then she has to face the 'Collapse Problem'; or she has to specify which kind of representational relation holds between the scientific theories and the world. The risk in this case is that there is not a fully realist account of the representational relation.

In a nutshell, a relation of isomorphism is a symmetric dyadic objective relation, while that of representation is an asymmetric 'triadic' intentional relation [37]. In other words, if we have to move beyond isomorphism we have to introduce a subject in our picture. For example, Giere argues for an 'agent-based' conception of representation, and describes it as composed by the following elements: "Agents (1) intend; (2) to use model, M; (3) to represent a part of the world, W; (4) for some purpose, P" $[38$, p. 269]. Giere also states that it is important to note "that this conception presupposes a notion of representation in general. I doubt that it is possible to give a noncircular (or reductive) account of representation in general" (Ibidem, p. 274). This means that we do not have a formal account of the notion of 'representation' which is 'objective' in the same way in which the formal account we have of the notion of isomorphism is 'objective'. Bas van Fraassen clearly states that, terminology aside, "a scientific model is a representation. So even if a scientific theory is a set of scientific models, and those literally are mathematical structures, it does not follow that the identity of a theory can be defined in terms of the corresponding set of mathematical structures without reference to their representational function" [39, p. 278].

The fact is that 'representation' is a semiotic relation: someone describes something as something else. There is an intrinsic subjective element in such an account which is unpalatable for many realists. A representation is subject- and contextdependent, while the realists aim at truth, and normally conceive of truth as mind- and stance-independent. Thus, if we maintain that we represent some phenomena, the problem is how to claim that our representation is the right representation, i.e. that it corresponds to reality. In other words, a satisfying realist account should concern only 
the model and the world, and should not include any reference to the knowing subject, while the notion of 'representation' seems to intrinsically involve a reference to the subject.

French is aware of the difficulty of solving such problem, but his answer is clearly unsatisfying. Indeed he simply acknowledges that the fact that:

\begin{abstract}
the relationship between any formal representation and the physical systems that it represents cannot be captured in terms of the former only [...] led to the accusation that the structuralist who relies on such representational devices cannot give an appropriate account of the relationship between representations and the world in terms of those very representations. My response is that all current forms of realism must face this accusation, not just OSR. ${ }^{7}$
\end{abstract}

This is clearly an unsatisfying answer, because the fact that to account for the relation between theories and the world is a problem for any kind of realism, does not diminish the relevance of such problem for OSR.

\title{
4.4 French's Approach to the Collapse Problem
}

French faces the above described difficulties claiming both that 1) the distinction between mathematics and the physical has to be accounted for in terms of causality: "how we can distinguish physical structure from mathematical structure [...]. The obvious answer is in terms of causality, with the physical structure we are to be realists about understood as fundamentally causal" [9, p. 166]; and that 2) we can secure the fact that our representation of the world is the right one relying on a sort of NMA: "In the realist case, we will have only the 'no miracles' intuition to justify our claim that our theories represent the structure of the world" [40, p. 57, fn 24].

These two claims can be reduced to one. In fact: how can we be sure that causality is a genuine feature of the world and not a feature of our model, as the anti-realists would suggest, and so that we can safely rely on causality to distinguish the physical from the mathematical? We can be sure that causality is a feature of the world only if we adopt a realist stance: "Ladyman follows Giere [...], who states that 'the crucial dividing line between empiricism and realism' concerns the status of modality, and urges that representing the world as modal is essential to the [...] realist" (Ibidem, p. 58). Thus, the way in which we ground the claim that a physical structure does not coincide with a mathematical structure is based on a sort of NMA exactly in the same way in which the claim that our representation of the world is the right representation is based on a sort of NMA. To better see this point, let's follow the argument given by French on a related issue:

On what basis can we ascribe lawhood [...] to the world? Here we need to articulate the ascription [...] within the structuralist framework: first of all, there is the attribution of laws, as features of structure, to the world. [...]. The structuralist can follow this line

7 [1, p. 195, fn 7]. 
and maintain a form of the No Miracles Argument, concluding that the best explanation for the success of a given theory is that 'its' laws are 'out there' in the world, as features of the structure of the world. ${ }^{8}$

Thus, the way to claim for a realist stance on causality is relying on a form of NMA. But the main appeal of SSR was exactly due to its supposed ability in avoiding the PMI, i.e. in supporting realism without directly relying on the NMA, given that the NMA is vulnerable to the PMI.

The fact is that if we accept OSR, we have to face the Collapse Problem. But, if we ground our defence from the Collapse Problem on causality and we justify our confidence in the fact that causality is a feature of the world and not a feature of the model relying on a sort of NMA, we find ourselves in the very same position in which the classical realists were in confronting the PMI. In fact, if we rely on the NMA, then we cannot be sure that a feature belongs to the world and not to the model, because we cannot exclude that our theory is false or incomplete, and so that the feature in question does not really correspond to anything in the world. If there is not a complete correspondence between our theory and the world, we are not able to safely state whether a feature belongs to the world or not. On the contrary, if a perfect correspondence holds, we can safely claim that everything that is in the model corresponds to something in the world. In this case we could safely assume that causality is in the world. But if we adopt OSR, to state a perfect correspondence between a theory and the world would amount to state that there is a relation of isomorphism between the mathematical structure of that theory and the world. But a relation of isomorphism may hold only between two mathematical structures. Thus, the Collapse Problem would step back again, and we would not be able to distinguish the mathematical and the physical anymore. Thus, we have to conclude that we are not able to certainly state whether causality is a feature of the world or not, and so that there is not an easy solution to the Collapse Problem for the realist based on causality. Both Psillos' and French's approaches seem to be inadequate.

\section{$5 \quad$ Structural Realism and Biology}

\subsection{Ontic Structural Realism and Price's Equation}

Let's now turn to French's proposal of adopting OSR in dealing with biology. French's attempt to articulate a biological form of OSR faces "the obvious problem of a comparative paucity of mathematized equations or laws by means of which we can identify and access the relevant structures" [1, p. 329]. But such paucity of mathematical structures does not affect "all biological fields- population genetics and theoretical ecology are the exceptions" (Ibidem, p. 329, fn 8). Thus, French focuses on Price's Equation, which is "sometimes presented as representing 'The Algebra of Evolution', and which one could take as characterizing a certain fundamental-if, perhaps, abstract—and 'high-level' feature of biological structure" (Ibidem, p. 338). Indeed,

$8 \quad$ [1, pp. 274-275]. 
Price's Equation is a central result in population genetics, and can be written in the following form:

$$
\Delta \mathrm{z}=\operatorname{Cov}(\mathrm{w}, \mathrm{z})+\operatorname{Ew}(\Delta \mathrm{z})
$$

where: ' $\Delta z$ ' is the change in average value of a character from one generation to the next; ' $\operatorname{Cov}(\mathrm{w}, \mathrm{z})$ ' represents the covariance between fitness $\mathrm{w}$ and character (action of selection); and ' $\operatorname{Ew}(\Delta \mathrm{z})$ ' represents the fitness weighted average of transmission bias. The equation "separates the change in average value of character into two components, one due to the action of selection, and the other due to the difference between offspring and parents" (Ibidem). According to French, there is a sense in which Price's Equation "offers a kind of 'meta-model' that represents the structure of selection in general [...] this covariance equation is independent of objects, rests on no contingent biological assumptions, and can be understood as representing the modal, relational structure of the evolutionary process" (Ibidem, italics mine).

\subsection{Fisher's Fundamental Theorem of Natural Selection}

In order to assess the claim made by French relative to Price's Equation, let's consider a special case of Price's Equation which has been widely debated: Fisher's Fundamental Theorem of Natural Selection (FTNS).

In fact, Price's Equation tells us exactly how much of a character will exist in the population in the next period. If we let the character equal fitness itself, then we get Fisher's theorem:

$$
\Delta \overline{\mathrm{w}}=\operatorname{Var}_{\mathrm{add}}(\mathrm{g}) / \overline{\mathrm{w}}
$$

which can be read as: the change in average fitness from one generation to another equals the additive genetic variance in the first generation, divided by mean fitness. The additive genetic variance, i.e. ' $\operatorname{Var}_{\text {add }}(\mathrm{g})$ ', measures the fitness variation in the population that is due to the additive, or independent, action of the genes. In other words, it measures any gene's effect on fitness which is independent of its genetic background. Indeed, according to this view of population genetics, it is possible to see the total 'Genetic Variance' as the sum of the 'Additive Genetic Variance' and the 'Non-additive Genetic Variance'.

Since its formulation, the FTNS has received different interpretations. This is due to the unclear formulation of the FTNS given by Fisher in his writings. In fact, Fisher describes the FTNS as follows: "the rate of increase in fitness of any organism at any time is equal to its genetic variance in fitness at that time" [41, p. 35].

This formulation of the FTNS induced many authors (and Fisher among them) to compare the FTNS to the second law of thermodynamics, according to which entropy, on average, can never decrease. In this interpretation, the FTNS is thought to be able to give a formal definition of the 'directionality of evolution', i.e. to give a proof of the fact that fitness, on average, will never decrease. Such a kind of result would have explained the course of evolution, the development of more and more complex forms, without any reference to any kind of 'design' or 'teleological explanation'. 
The problem is that "it is simply untrue that the average fitness of a population undergoing natural selection never decreases, so the rate of change of average fitness cannot always be given by the additive genetic variance" [42, p. 328]. Okasha clarifies that "Fisher was not talking about the rate of change of average fitness at all, but rather the partial rate of change which results from [the direct action of] natural selection altering gene frequencies in the population, in a constant environment" (Ibidem, p. 329). ${ }^{9}$

This means that it is more careful to say that according to the FTNS when natural selection is the only force in operation, average fitness can never decrease.

\subsection{The Failure of the Analogy with Thermodynamics}

The problem with this more careful interpretation of the FTNS is that it undermines the analogy between the FTNS and the second law of thermodynamics. Indeed, "by Fisher's lights, natural selection will almost never be the only force in operation; for by causing gene frequencies to change, selection almost always induces environmental change, which is itself a force affecting average fitness" (Ibidem, p. 344). In fact "the environment in Fisher's sense will not remain fixed, for selection itself alters it" (Ibidem, p. 347). Details are not relevant here, the basic idea is that for Fisher, when natural selection operates, this fact directly alters both the mean fitness $\overline{\mathrm{w}}$, and the 'environment', which at its turn alters the mean fitness $\overline{\mathrm{w}}$. Thus, if the FTNS holds only when natural selection is the only force to operate in a constant environment, and if when natural selection operates, the environment cannot remain constant, then we should conclude that the situation described by the FTNS can never obtain. Thus, the analogy between fitness and entropy seems to fail.

\subsection{Different Interpretations of the Fundamental Theorem}

The biological meaning of the FTNS is at least contentious. Price [43] and Ewens [44] state that the FTNS is mathematically correct, but that it does not have the biological significance that Fisher claimed for it. On the contrary, Edwards [45] and Grafen [46] are much more sympathetic to Fisher. We cannot develop this issue here. What we will analyse is whether French's proposal may be useful in dealing with this topic. In other words, can OSR help us in trying to determine the biological significance of the FTNS?

The fact is that both the main interpretations of the FTNS agree on the mathematical validity of Fisher's result. Thus, since structuralism seems to be mainly committed

\footnotetext{
9 To understand Fisher's understanding of the FTNS, we have to accept Fisher's view of 'environment': any change in the average effects constitutes an 'environmental' change. On the constancy of environment, cf. [42, p. 331]: "For Fisher, constancy of environment from one generation to another meant constancy of the average effects of all the alleles in the population. Recall that an allele's average effect (on fitness) is the partial regression of organismic fitness on the number of copies of that allele." Cf., also, Ibidem, p. 324: "an allele's average effect may change across generations, for it will often depend on the population's genetic composition."
} 
to the mathematical structures of a theory, and since the two main interpretations of the FTNS do not diverge on this issue, OSR seems prima facie unable to asses which interpretation of the FTNS is to be preferred. But French's formulation of OSR states that structures have to be interpreted in causal terms, in order to face the Collapse Problem. Thus, it seems that French's approach should be able to assess which interpretation of the FTNS we should prefer. Indeed, the issue of the interpretation of the FTNS is deeply related to the issue of the nature of natural selection, i.e. to the debate over the causal nature of natural selection.

\subsection{The Fundamental Theorem and the Nature of Natural Selection}

Let's restate the issue we are dealing with: French supports the claim that Price's Equation gives us the modal structure of the evolutionary process. The FTNS is a special case of Price's Equation. Thus, we can infer that French supports an interpretation of the FTNS as a significant and substantial result referring to natural selection. Since French claims also that structures have to be interpreted as causal in order to avoid the Collapse Problem, and since, as we have seen, he seems to support the biological significance of the FTNS, we should infer that he thinks that the relevant mathematical structure in this context, i.e. the FTNS, can be interpreted in causal terms, i.e. that the FTNS can be interpreted as referring to some causal process.

Now, the problem is that the process to which the FTNS refers is that of natural selection, and that the causal nature of such process is harshly debated. It is not relevant to take side on this issue here. What is worth underling is that, contrary to French's proposal, those who deny the causal nature of natural selection explicitly refer to the FTNS and its mathematical formulation and significance to support their claim that such a kind of result cannot be interpreted as referring to causal processes.

\subsection{The Causal Nature of Natural Selection}

The causal nature of natural selection has recently been put under severe scrutiny. ${ }^{10}$ This sort of "causal scepticism is motivated by the fact that most, if not all, principles of evolutionary theory - such as the Price equation or Fisher's fundamental theorem of natural selection - are expressed by purely statistical terms such as variances or covariances" [47, p. 2]. For example, Matthen and Ariew state that the reason "for reifying natural selection $[. .$.$] lies in \mathrm{a}[\mathrm{n}][\ldots]$ analogy between equations of population genetics - such as Fisher's Theorem - and certain equations of physics" [48, p. 208]. But this analogy is not well founded, because, unlike the models that we find in physics, the descriptions of natural selection "rendered by population genetics models are in general neither predictive nor explanatory," since "population genetics models are, in general, noncausal models" [49, pp. 369, 383]. Moreover, natural selection itself is not a genuine feature of the world, it is just "ontologically derivative on individual-level events such as births, deaths, and mutations" [48, p. 216].

${ }^{10}$ See [47] for a survey. 
This view of the nature of natural selection seems to conflict with French's idea that the mathematical structures of a theory have to be intended as causal and that mathematical structures give us the fundamental structures of the world. Indeed, it would be difficult to accept that we should think that the FTNS tells us something about the deep (causal) structure of the evolutionary process, i.e. the core process of biology, if such theorem refers to something which is not only an 'ontologically derivative' non-causal concept, but which is also not intrinsically related to anything biological in nature. For example, Matthen and Ariew state that natural selection "is not even a biological phenomenon as such. It holds in any history in which the terms of the theory can be jointly interpreted in a way that accords with the abstract requirements of the theory" (Ibidem, p. 222). To illustrate this point they show how the FTNS may be equally well applied to something which is certainly not a biological entity or process:

Suppose that you have two bank accounts, one yielding $5 \%$ interest and the other yielding 3\%. One can treat units of money in each account as the members of a population, and the interest rate as an analogue of fitness. Provided that no money is transferred from one account to another, one can treat these 'fitness' values as heritable - that is, the fitness of any particular (non original) piece or unit of money can be ascribed to the 'reproductive' rate (i.e., interest) on preexisting units of money. Thus you would have, as between the monies resident in the two accounts, variation in heritable fitness. On this interpretation, Fisher's Fundamental Theorem of Natural Selection applies to your bank accounts: it predicts (correctly) that the average interest earned by the two bank accounts taken together will increase in proportion to the variance of interest rates earned by your money in the two accounts. ${ }^{11}$

As already noted, here the issue is not taking side on the dispute over the nature of natural selection, but underling how the difficulty of assessing such nature poses a challenge for French's proposal.

\subsection{Ontic Structural Realism and the Meaning of Price's Equation}

The fact is that French seems to acknowledge the abstract and not-intrinsically biological character of Price's Equation. For example, he states that "Price himself emphasized that his equation could be used to describe the selection of radio stations with the turning of a dial just as easily as it could to describe biological evolution" [1, p. 338 , fn 8]. Even if we accept, for the sake of the argument, that there could be a way to make this abstractness compatible with the claim that Price's Equation gives us the deep structure of biological evolution, there remains a problem. The problem is that several authors support the idea that it is possible to give a causal interpretation of the FTNS [50-51]. Indeed, the causal interpretation of the evolutionary principles "shows adaptive evolution as a genuine causal process, where fitness and selection are both causes of evolution" [47, p.1]. But French does not refer to such authors. He instead

11 [48, p. 222]. 
explicitly refers several times to Samir Okasha's works [1, Ch. 12]. The point is that Okasha does not adopt a straightforward causal interpretation of the fundamental results of population genetics. For example, he states that "Price's equation is statistical not causal" [52, p. 25]. Even more explicitly, Okasha states that Price's Equation "is simply a mathematical tautology whose truth follows from the definition of the terms. Nothing is assumed about the nature of the 'entities', their mode of reproduction, the mechanisms of inheritance, the genetic basis of the character, or anything else" (Ibidem, p. 24).

Thus, the problem is that French has not defended at all the claim that natural selection has a causal nature. The only mathematical structure taken into account by French, i.e. Price's Equation, which is supposed to be able to give us the deep structure of the evolutionary process, gives raise (mainly) to two different and incompatible interpretations. But French's structural proposal could help us in solving such issue only if the dispute over the causal nature of natural selection would have been already settled in favor of the causalists, and French says nothing on this point.

\section{Conclusions}

To conclude, let's briefly sum up the difficulties that French's proposal of adopting OSR in biology has to face, in order to assess whether this proposal gives us some advantage in philosophically dealing with biology. French seems to accept the causal nature of natural selection and the idea that structures have to be understood as causal structures, but Price's Equation and other population genetics results, as the FTNS, are often interpreted as giving non-causal explanations, also by those authors to whom French himself refers in order to illustrate his proposal. In focusing on the mathematical abstract features of such equations, French seems to think that such structural characteristics are enough to give us a structural description of biology. But then there is nothing which can help us in avoiding the Collapse Problem according to the French's own strategy to face this problem: there is nothing in French's proposal on Price's Equation which suggests that 1) the causal nature of natural selection can be safely shown to be a feature of the world and that 2) such feature of the world is correctly reflected by the population genetics models. Only if 1) and 2) obtain, in fact, the abstract structure given by Price's Equation could be interpreted both as the fundamental structure of biology and a causal structure. On the contrary, we have shown that there are relevant difficulties in showing that both these conditions hold.

Moreover, we have to stress that French's proposal does not confront at all with the traditional semanticist approach to biology, which claims that it is necessary to give a phase space of the biological system we are modeling. French contents himself with referring only to some equations, and so he neither defends the possibility of giving a semanticist account without having to give the phase space, nor tries to give a complete phase space of the model he is considering. He explicitly states: "How are we to identify these structures that we are supposed to be realist about? The most obvious route is via the equations" $[9$, p. 165]. But, as we have seen, this is an insufficient 


\section{PENULTIMATE DRAFT - PLEASE CITE THE PUBLISHED VERSION}

To appear in: Models and Inferences in Science, Ippoliti, E., Sterpetti, F. and Nickles, T. (eds.), Springer.

response to the detailed remarks made by Gildenhuys on the difficulty of constructing a phase space when dealing with population genetics.

Thus, it seems reasonable to conclude that in the biological domain, OSR has to face the same challenges that it has to face in other domains, and that dealing with biological issues does not give to OSR any peculiar help in facing those challenges, e.g. the Collapse Problem. At the same time, OSR seems not able to solve any peculiar philosophical issue related to population genetics, since, on the contrary, it is the solution of a debated philosophical issue related to biology, such that of the nature of the natural selection, which, if given, could represent a support to a structuralist approach to population genetics.

\section{References}

1. French, S.: The Structure of the World. Oxford University Press, Oxford (2014)

2. Saatsi, J., Vickers, P.: Miraculous Success? Inconsistency and Untruth in Kirchhoff's Diffraction Theory. The British Journal for the Philosophy of Science 62, 29-46 (2011)

3. Ellis, B.: The Metaphysics of Scientific Realism. Acumen, Durham (2009)

4. Giere, R.N.: Scientific Realism: Old and New Problems. Erkenntnis 63, 149-165 (2005)

5. Sankey, H.: Scientific Realism and the Rationality of Science. Ashgate, Burlington (2008)

6. Putnam, H.: Mathematics, Matter and Method. Cambridge University Press, Cambridge (1975)

7. Laudan, L.: A Confutation of Convergent Realism. Philosophy of Science 48, 19-49 (1981)

8. Magnus, P.D.: Inductions, Red Herrings, and the Best Explanation for the Mixed Record of Science. The British Journal for the Philosophy of Science 61, 803-819 (2010)

9. French, S.: Shifting to Structures in Physics and Biology: A Prophylactic for Promiscuous Realism. Studies in History and Philosophy of Biological and Biomedical Sciences 42, 164-173 (2011)

10. French, S., Ladyman, J.: Remodelling Structural Realism: Quantum Physics and the Metaphysics of Structure. Synthese 136, 31-56 (2003)

11. Worrall, J.: Structural Realism: The Best of Both Worlds? Dialectica 43, 99-124 (1989)

12. Ladyman, J. What Is Structural Realism? Studies in History and Philosophy of Science 29, 409-424 (1998)

13. Frigg, R., Votsis, I.: Everything You Always Wanted to Know about Structural Realism but Were Afraid to Ask. European Journal for Philosophy of Science 1, 227-276 (2011)

14. Sneed, J.: Structuralism and Scientific Realism. Erkenntnis 19, 345-370 (1983)

15. Chakravartty, A.: The Semantic or Model-Theoretic View of Theories and Scientific Realism. Synthese 127, 325-345 (2001)

16. Halvorson, H.: What Scientific Theories Could Not Be. Philosophy of Science 79, 183$206(2012)$

17. da Costa, N.C.A., French, S.: Science and Partial Truth. A Unitary Approach to Models and Scientific Reasoning. Oxford University Press, Oxford (2003)

18. Suppe, F.: Understanding Scientific Theories: An Assessment of Developments, 19691998. Philosophy of Science 67, S102-S115 (2000)

19. Steiner, M.: The Applicability of Mathematics as a Philosophical Problem. Harvard University Press, Cambridge (MA) (1998)

20. Tegmark, M.: The Mathematical Universe. Foundations of Physics 38, 101-150 (2008) 


\section{PENULTIMATE DRAFT - PLEASE CITE THE PUBLISHED VERSION}

To appear in: Models and Inferences in Science, Ippoliti, E., Sterpetti, F. and Nickles, T. (eds.), Springer.

21. Lloyd, E.A.: A Semantic Approach to the Structure of Population Genetics. Philosophy of Science 51, 242-264 (1984)

22. Thompson, P.: The Structure of Evolutionary Theory: A Semantic Approach. Studies in History and Philosophy of Science 14, 215-229 (1983)

23. Beatty, J.: Optimal-Design Models and the Strategy of Model Building in Evolutionary Biology. Philosophy of Science 47, 532-561 (1980)

24. Beatty, J.: The Evolutionary Contingency Thesis. In: Wolters, G., Lennox, J.G. (eds.) Concepts, Theories, and Rationality in the Biological Sciences, pp. 45-81. University of Pittsburgh Press, Pittsburgh (1995)

25. French, S., Saatsi, J.: Realism about Structure: The Semantic View and Nonlinguistic Representations. Philosophy of Science 73, 548-559 (2006)

26. van Fraassen, B.C.: Scientific Representation. Oxford University Press, Oxford (2008)

27. Gildenhuys, P.: Classical Population Genetics and the Semantic Approach to Scientific Theories. Synthese 190, 273-291 (2013)

28. Lloyd, E.A.: The Structure and Confirmation of Evolutionary Theory. Princeton University Press, Princeton (1994)

29. Longo, G., Montévil, M.: Perspectives on Organisms. Biological Time, Symmetries and Singularities. Springer, Berlin (2014)

30. Psillos, S.: Anti-Nominalistic Scientific Realism: A Defense. In: Bird, A., Ellis, B., Sankey, H. (eds.) Properties, Powers and Structures. Issues in the Metaphysics of Realism, pp. 63-80. Routledge, New York (2012)

31. Rice, C.: Moving Beyond Causes: Optimality Models and Scientific Explanation. Noûs, DOI: 10.1111/nous.12042 (2013)

32. Psillos, S.: Living with the Abstract: Realism and Models. Synthese 180, 3-17 (2011)

33. Newstead, A., Franklin, J.: Indispensability without Platonism. In: Bird, A., Ellis, B., Sankey, H. (eds.) Properties, Powers and Structures. Issues in the Metaphysics of Realism, pp. 81-97. Routledge, New York (2012)

34. Balaguer, M.: Realism and Anti-Realism in Mathematics. In: Gabbay, D., Thagard, P., Woods, J. (eds.) Handbook of the Philosophy of Science. Volume 4. Philosophy of Mathematics, pp. 117-151. Elsevier, Amsterdam (2009)

35. Psillos, S.: The Scope and Limits of the No Miracles Argument. In: Dieks, D., Gonzalez, W.J., Hartmann, S., Uebel, T., Weber, M. (eds.) Explanation, Prediction, and Confirmation, pp. 23-35. Springer, Dordrecht (2011)

36. Hughes, R.I.G.: Models, the Brownian Motion, and the Disunities of Physics. In: Earman, J., Norton, J. (eds.) The Cosmos of Science, pp. 325-347. University of Pittsburgh Press, Pittsburgh (1997)

37. Suárez, M.: Scientific Representation: Against Similarity and Isomorphism. International Studies in the Philosophy of Science 17, 225-244 (2003)

38. Giere, R.N.: An Agent-Based Conception of Models and Scientific Representation. Synthese 172, 269-281 (2010)

39. van Fraassen, B.C.: One or Two Gentle Remarks about Hans Halvorson's Critique of the Semantic View. Philosophy of Science 81, 276-283 (2014)

40. Brading, K.: Structuralist Approaches to Physics: Objects, Models and Modality. In: Bokulich, A., Bokulich, P. (eds.) Scientific Structuralism, pp. 43-65. Springer, Dordrecht (2011)

41. Fisher, R.A.: The Genetical Theory of Natural Selection. Clarendon Press, Oxford (1930)

42. Okasha, S.: Fisher's Fundamental Theorem of Natural Selection - A Philosophical Analysis. The British Journal for the Philosophy of Science 59, 319-351 (2008) 


\section{PENULTIMATE DRAFT - PLEASE CITE THE PUBLISHED VERSION}

To appear in: Models and Inferences in Science, Ippoliti, E., Sterpetti, F. and Nickles, T. (eds.), Springer.

43. Price, G.R.: Fisher's 'Fundamental Theorem' Made Clear. Annals of Human Genetics 36, 129-140 (1972)

44. Ewens, W.J.: An Interpretation and Proof of the Fundamental Theorem of Natural Selection. Theoretical Population Biology 36, 167-180 (1989)

45. Edwards, A.W.F.: The Fundamental Theorem of Natural Selection. Biological Review 69, 443-474 (1994)

46. Grafen, A.: Fisher the Evolutionary Biologist. The Statistician 52, 319-329 (2003)

47. Otsuka, J.: Causal Foundations of Evolutionary Genetics. The British Journal for the Philosophy of Science, DOI: 10.1093/bjps/axu039 (2014)

48. Matthen, M., Ariew, A.: Selection and Causation. Philosophy of Science 76, 201-224 (2009)

49. Glymour, B.: Wayward Modeling: Population Genetics and Natural Selection. Philosophy of Science 73, 369-389 (2006)

50. Sober, E.: The Nature of Selection. University of Chicago Press, Chicago (1984)

51. Millstein, R.: Natural Selection as a Population-Level Causal Process. The British Journal for the Philosophy of Science 57, 627-653 (2006)

52. Okasha, S.: Evolution and the Levels of Selection. Oxford University Press, Oxford (2006) 